\title{
Sky coverage estimates for adaptive optics systems from computations in Zernike space
}

\author{
Richard M. Clare \\ Thirty Meter Telescope Project, 1200 East California Boulevard, Mail Code 102-8, Pasadena, California 91125, and \\ Association of Universities for Research in Astronomy New Initiatives Office, 950 North Cherry Avenue, \\ Tucson, Arizona 85719 \\ Brent L. Ellerbroek \\ Thirty Meter Telescope Project, 1200 East California Boulevard, Mail Code 102-8, Pasadena, California 91125
}

Received May 19, 2005; accepted July 26, 2005

\begin{abstract}
A sky coverage model for laser guide star adaptive optics systems is proposed. The atmosphere is considered to consist of a finite number of phase screens, which are defined by Zernike basis polynomials, located at different altitudes. These phase screens are transformed to the aperture plane, where they are converted to laser and natural guide star wavefront sensing measurements. These transformations incorporate the cone effect due to guide stars at finite heights, anisoplanatism due to guide stars off axis with respect to the science object, and adaptive optics systems with multiple guide stars. The wavefront error is calculated tomographically with minimum variance estimators derived from the transformation matrices and the known statistical properties of the atmosphere. This sky coverage model provides fast Monte Carlo simulations over random natural guide star configurations, irrespective of telescope diameter. The Monte Carlo simulations outlined show that inclusion of a finite outer scale for the atmosphere significantly reduces the median wavefront error, that increasing the number of laser guide stars in the asterism reduces the median wavefront error, and that a larger natural guide star patrol field provides a smaller median wavefront error when there is a low star density in the field. (C) 2006 Optical Society of America
\end{abstract}

OCIS codes: $010.1080,010.7350$.

\section{INTRODUCTION}

The resolution of ground-based telescopes is limited by the wavefront aberrations induced by the earth's atmosphere. ${ }^{1}$ Babcock proposed overcoming these wavefront aberrations in real time by placing a correcting element in the optical path, ${ }^{2}$ in a system that has become known as adaptive optics (AO). AO remains critical to the successful operation of the next generation of groundbased telescopes of diameter 30-100 m. ${ }^{3,4}$

Current AO systems ${ }^{5}$ employ sodium laser guide stars (LGSs) to increase the signal-to-noise ratio for the wavefront sensors that measure the instantaneous wavefront aberrations. LGS AO, however, has two major inherent difficulties. First, LGSs suffer from the so-called "cone effect" because the beacon is located at a finite height (approximately $90 \mathrm{~km}$ ) and can only sample the cone of atmosphere below it. ${ }^{6}$ Second, the LGSs cannot measure the tip/tilt (TT) modes because the laser is equally deflected on the up and down paths and also because practical considerations limit the precision of laser pointing.

Multiconjugate adaptive optics (MCAO), where there are multiple deformable mirrors (DMs) conjugate to different altitudes in the atmosphere, has been proposed to overcome the cone effect and provide imaging over a wider field of view (FOV). ${ }^{7}$ Multiple LGSs are used to tomographically reconstruct atmospheric turbulence in three dimensions and determine the commands for the multiple DMs. An alternative AO configuration for tomography and wide-field correction is multiobject adaptive optics (MOAO), where the DMs apply independent corrections to objects within the FOV. MOAO is a generalization of the FALCON approach. ${ }^{8}$ Like MCAO, MOAO uses multiple GSs to tomographically reconstruct atmospheric turbulence. The difference between MCAO and MOAO is that in MCAO the DMs operate in series and in MOAO the DMs operate in parallel.

Even multiple LGSs cannot, however, measure the TT from the atmosphere, which are the largest terms in the uncorrected wavefront. ${ }^{9}$ It is therefore necessary to also employ natural guide star (NGS) wavefront sensors to estimate these modes. Yet there is no guarantee that a NGS that is bright enough to drive the wavefront sensor will exist within the isoplanatic patch for a given science object. Estimating the sky coverage that a LGS AO or MCAO system can attain is therefore essential to its design. In this paper, we consider a $30 \mathrm{~m}$ diameter telescope, although the methodology is valid for any telescope diameter.

When a TT NGS is viewed at a different angle from the science object (off axis), tilt anisoplanatism is induced into the wavefront. Tilt anisoplanatism is the fundamental effect limiting sky coverage for conventional singleconjugate LGS AO systems. For LGS MCAO, tilt anisoplanatism will still degrade the uniformity of turbulence compensation over an extended field if a single TT NGS is available, since the so-called null modes ${ }^{10}$ responsible for tilt anisoplanatism cannot be inferred from the LGS wavefront sensing (WFS) measurements alone. An 
example of such a null mode is a positive focus at one altitude and a negative focus at another. A number of strategies have been proposed to overcome this problem including GSs at different altitudes (i.e., a combination of Rayleigh and sodium LGSs), multiple TT NGSs, and a NGS that measures tip/tilt/focus/astigmatism (TTFA). ${ }^{11}$ We consider the latter two approaches in this paper, although the methodology is also valid for the first.

In this paper, we will outline a novel method for estimating the sky coverage that takes into account the tilt anisoplanatism caused by off-axis GSs, the cone effect from GSs located at finite altitudes, and the tomographic reconstruction of turbulence in MCAO and MOAO systems. The essence of this method is to project the phase screens, which are represented as a Zernike basis sum, at each turbulent layer of the atmosphere to the telescope aperture plane, where they are converted to WFS measurements. We then use the known statistical properties of the atmosphere to calculate the wavefront error with a minimum variance estimator. This process is repeated over random NGS constellations to give an estimate of the wavefront error as a function of NGS density. Working in Zernike space enables results to be obtained rapidly, since only the lower order modes of the wavefront need to be considered to assess sky coverage, regardless of telescope aperture diameter.

The remainder of this paper is structured as follows. In Section 2, the mathematical framework for this paper is outlined. In particular, the Zernike basis function representation of the wavefronts, the transformation matrices, and optimal wavefront estimators are discussed. The Monte Carlo simulations of sky coverage with random NGS configurations are presented in Section 3. Conclusions from this research are drawn in Section 4.

\section{WAVEFRONT ESTIMATION}

The following analysis assumes geometrical optics, such that phase errors are accumulated over a ray path. ${ }^{12}$

\section{A. Basis Polynomials}

We consider the atmospheric turbulence to consist of a finite number of layers, $N_{l}$, of phase screens located at heights $h(i)$. We describe each phase screen in terms of the Zernike polynomial basis functions, ${ }^{9}$ which are orthonormal over the unit circle and whose low order terms represent the classical optical aberrations of tilt, focus, astigmatism, etc. The phase screen located at the $i$ th layer, $\phi(i ; r, \theta)$, is completely described by

$$
\phi(i ; r, \theta)=\sum_{n=0}^{\infty} \sum_{m=0}^{n} a_{n m}(i) Z_{n m}(r, \theta),
$$

where $Z_{n m}$ is the $m$ th Zernike polynomial of radial order $n$ and $a_{n m}$ are the coefficients of the corresponding Zernike polynomials.

It is, however, also convenient to describe the phase screens as monomials in order to deal with the off-axis projection and finite range of the GS, and we follow the method of Lloyd-Hart and Milton ${ }^{12}$ and Ragazzoni et al. ${ }^{13}$ in using rectangular coordinates to define the phase screens. The phase screens can therefore be defined by ${ }^{12}$

$$
\begin{aligned}
\phi(i ; x, y) & =\phi(i ; r, \theta) \approx \sum_{n=0}^{N} \sum_{m=0}^{n} a_{n m}(i) Z_{n m}(r, \theta) \\
& =\sum_{j=0}^{N} \sum_{k=0}^{N-j} \alpha_{j k}(i) x^{j} y^{k},
\end{aligned}
$$

where a finite $N$ radial orders of Zernikes are used and $(x, y)$ are the coordinates in the plane of the phase screen.

$Q$ is the matrix that transforms the Zernike coefficients in polar coordinates $a$ to monomials in rectangular coordinates $\alpha$, such that

$$
\alpha=Q a \text {. }
$$

$Q$ is square and nonsingular. ${ }^{12}$ If there are multiple layers of turbulence, $Q$ is a block diagonal matrix of the form

$$
Q=\left[\begin{array}{ccccc}
Q(1) & & & & 0 \\
& \ddots & & & \\
& & Q(i) & & \\
& & & \ddots & \\
0 & & & & Q\left(N_{l}\right)
\end{array}\right],
$$

where the $Q(i)$ will be identical if the same order of Zernike polynomials is used for each atmospheric layer.

Similarly,

$$
\alpha=\left(\begin{array}{c}
\alpha_{j k}(1) \\
\vdots \\
\alpha_{j k}(i) \\
\vdots \\
\alpha_{j k}\left(N_{l}\right)
\end{array}\right),
$$

$$
a=\left(\begin{array}{c}
a_{n m}(1) \\
\vdots \\
a_{n m}(i) \\
\vdots \\
a_{n m}\left(N_{l}\right)
\end{array}\right),
$$

\section{B. Transformation Matrices}

We transform the phase screens located at heights $h(i)$ to the aperture plane by applying the transformation matrix $T$ :

$$
b=T a,
$$

where $b$ is the vector of Zernike coefficients of the phase aberration or aberrations projected to the aperture from one of several GSs and $a$ is as defined in Eq. (6). The transformation matrix is defined by

$$
T=Q^{-1} S C Q,
$$

where $S$ and $C$ take into account the shifting due to offaxis projection of the $l$ th GS at an angular position $\left(\theta_{x}(l), \theta_{y}(l)\right)$ and the cone effect due to the finite altitude $H$ of the LGS, respectively. The summation over the different layers of the atmosphere is performed inside the matrix $S$. As mentioned in Subsection 2.A, it is more convenient to deal with the off-axis GS and finite GS altitude in 
monomial space than in Zernike space, so the Zernike coefficients are first converted to monomial coefficients by $Q$. After the cone effect and shifting operators have been applied, the monomial coefficients are converted back to Zernike coefficients by application of $Q^{-1}$.

For a LGS at an altitude $H$, Eq. (2) is scaled to account for the cone effect:

$$
\begin{gathered}
\left.\phi\left(i ; 1-\frac{h(i)}{H}\right] x,\left[1-\frac{h(i)}{H}\right] y\right) \\
=\sum_{j=0}^{N} \sum_{k=0}^{N-j} \alpha_{j k}(i) x^{j} y^{k}\left[1-\frac{h(i)}{H}\right]^{j+k} \\
=\sum_{j=0}^{N} \sum_{k=0}^{N-j}[C(i) \alpha(i)]_{j k} x^{j} y^{k},
\end{gathered}
$$

where $C(i)$, the cone effect operator on the $i$ th layer, is given by

$$
[C(i) \alpha(i)]_{j k}=\left[1-\frac{h(i)}{H}\right]^{j+k} \alpha_{j k}(i) .
$$

$C$ is a block diagonal matrix of the form

$$
C=\left[\begin{array}{ccccc}
C(1) & & & & 0 \\
& \ddots & & & \\
& & C(i) & & \\
& & & \ddots & \\
0 & & & & C\left(N_{l}\right)
\end{array}\right],
$$

where there are $i=1, \ldots, N_{l}$ layers of turbulence. Only sodium LGSs at one altitude, $H$, are considered in this paper, although it is possible to extend this method to include both Rayleigh and sodium LGSs.

We now consider the shifting due to the $l$ th GS at angular position $\left(\theta_{x}(l), \theta_{y}(l)\right)$ in the field. Phase aberrations at the $i$ th layer with coordinates $\left(x^{\prime}, y^{\prime}\right)$ are projected to the aperture with coordinates $(x, y)$ by ${ }^{14}$

$$
\begin{aligned}
& x^{\prime}=h(i) \theta_{x}(l)+\left[1-\frac{h(i)}{H}\right] x, \\
& y^{\prime}=h(i) \theta_{y}(l)+\left[1-\frac{h(i)}{H}\right] y .
\end{aligned}
$$

The wavefront aberration at the aperture caused from the $i$ th layer of turbulence sampled from the $l$ th GS is therefore given by substituting Eq. (13) into Eq. (2):

$$
\begin{aligned}
\phi\left(i, l ; h(i) \theta_{x}(l)+\left[1-\frac{h(i)}{H}\right] x, h(i) \theta_{y}(l)+\left[1-\frac{h(i)}{H}\right] y\right) \\
=\sum_{j=0}^{N} \sum_{k=0}^{N-j} \alpha_{j k}(i)\left\{h(i) \theta_{x}(l)+\left[1-\frac{h(i)}{H}\right] x\right\}^{j} \\
\times\left\{h(i) \theta_{y}(l)+\left[1-\frac{h(i)}{H}\right] y\right\}^{k} .
\end{aligned}
$$

We can expand Eq. (15) in a similar manner to Lloyd-Hart and Milton ${ }^{12}$ by using a binomial expansion, yielding

$$
\begin{gathered}
\phi\left(i, l ; h(i) \theta_{x}(l)+\left[1-\frac{h(i)}{H}\right] x, h(i) \theta_{y}(l)+\left[1-\frac{h(i)}{H}\right] y\right) \\
=\sum_{j=0}^{N} \sum_{k=0}^{N-j} \sum_{j^{\prime}=0}^{j} \sum_{k^{\prime}=0}^{k} \alpha_{j k}(i)\left[1-\frac{h(i)}{H}\right]^{j}\left[1-\frac{h(i)}{H}\right]^{k} \\
\times\left[\frac{h(i) \theta_{k}(l)}{h(i)}\right]^{j-j^{\prime}}\left[\frac{h(i) \theta_{y}(l)}{h(i)}\right]^{k-k^{\prime}}\left(\begin{array}{l}
j \\
j^{\prime}
\end{array}\right) x^{j^{\prime}}\left(\begin{array}{c}
k \\
k^{\prime}
\end{array}\right) y^{k^{\prime}} \\
\left.1-\frac{h}{H}\right] \\
=\sum_{j=0}^{N} \sum_{k=0}^{N-j} \sum_{j^{\prime}=0}^{j} \sum_{k^{\prime}=0}^{k}[C(i) \alpha(i)]_{j k}[S(l, i)]_{j^{\prime} k^{\prime}, j k} x^{j^{\prime}} y^{k^{\prime}}
\end{gathered}
$$

where

$$
[S(l, i)]_{j^{\prime} k^{\prime}, j k}=\left(\begin{array}{l}
j \\
j^{\prime}
\end{array}\right)\left(\begin{array}{c}
k \\
k^{\prime}
\end{array}\right)\left[\frac{h(i) \theta_{x}(l)}{1-\frac{h(i)}{H}}\right]^{j-j^{\prime}}\left[\frac{h(i) \theta_{y}(l)}{1-\frac{h(i)}{H}}\right]^{k-k^{\prime}}
$$

and $\left(\begin{array}{c}k \\ k^{\prime}\end{array}\right)$ is the binomial coefficient $k ! /\left[k^{\prime} !\left(k-k^{\prime}\right) !\right] . S(l, i)$ is the block of the shifting matrix due to the $l$ th GS propagating through the $i$ th layer of turbulence. The shifting matrix $S$ is not block diagonal and is of the form

$$
S=\left[\begin{array}{ccccc}
S(1,1) & S(1,2) & \ldots & & S\left(1, N_{l}\right) \\
S(2,1) & \ddots & & & \\
\vdots & & S(l, i) & & \\
& & & \ddots & \\
S\left(N_{G S}, 1\right) & & \ldots & & S\left(N_{G S}, N_{l}\right)
\end{array}\right]
$$

where there are $i=1, \ldots, N_{l}$ layers of turbulence and $l$ $=1, \ldots, N_{G S}$ guide stars. By choice of this definition for $S$, the summation of phase aberrations over all the layers of the turbulence occurs in $S$.

\section{Minimum Variance Wavefront Reconstruction}

In this subsection, expressions for the residual wavefront phase variances for both the MOAO and MCAO modes of operation, in terms of the transformation matrices $T$ and the covariance matrix of the atmosphere, $C_{\phi}$, are derived. As defined in Section 1, MCAO consists of one or more DMs that are conjugate to one or more layers of turbulence in the atmosphere, and MOAO consists of one or more DMs that correct one or more objects in the science field. The residual wavefront variances are derived by using a minimum variance estimator following the procedure of Fusco et al. ${ }^{15}$

First, the Zernike coefficients of the science phase at the aperture, $b_{s}$, are found by multiplying the coefficients at each altitude, $a$, by the science transformation matrix $T_{s}$ :

$$
b_{s}=T_{s} a .
$$

The coefficients $b_{s}$ describe the science wavefronts $\phi_{k}$ at the $k=1, \ldots, N_{s}$ points in the science field. 

by

The wavefront modes as seen by the LGS, $b_{L}$, are given

$$
b_{L}=T_{L} a .
$$

The LGSs are unable to estimate the TT modes because of the position uncertainty, so the first order radial modes are dropped from $b_{L}$.

Similarly, the wavefront modes as seen by the NGS, $b_{n}$, are given by

$$
b_{n}=T_{n} a .
$$

The number of Zernike modes constituting $b_{n}$ is generally small because the NGSs are assumed to be faint. In this paper, two regimes of NGS operation are considered: The NGS estimates the TT modes only, or the NGS estimates the TTFA modes only. Note that the effects of additive measurement noise and time delay are not yet included in these measurement models.

Finally, $b_{m}$ is the vector of phase corrections applied by the $M \mathrm{DM}$ to the science wavefronts based on the phase estimate $\hat{a}$, projected to the aperture plane, and is given by

$$
b_{m}=T_{m} m=T_{m} P_{m} \hat{a},
$$

where $m=P_{m} \hat{a}$ are the Zernike coefficients applied to the DMs and $P_{m}$ is the optimal fit of Zernike modes to the DMs to compensate the estimated phase profile $\hat{a}$.

The estimation problem here is to estimate the wavefront modes $\hat{a}$ from the wavefront modes observed with the LGS, $b_{L}$, and NGS, $b_{n}$, respectively. The minimum variance estimator is given by ${ }^{16}$

$$
\hat{a}=\left\langle a\left(b_{L}{ }^{\mathrm{T}} \quad b_{n}{ }^{\mathrm{T}}\right)\right\rangle\left\langle\left(\begin{array}{l}
b_{L} \\
b_{n}
\end{array}\right)\left(b_{L}{ }^{\mathrm{T}} b_{n}{ }^{\mathrm{T}}\right)\right\rangle^{\dagger}\left(\begin{array}{l}
b_{L} \\
b_{n}
\end{array}\right),
$$

where $\dagger$ is the pseudoinverse operator, which in this paper is computed with the singular value decomposition and $\langle\cdot\rangle$ denotes an ensemble average. Substitution of Eqs. (21) and (22) into Eq. (24) yields

$$
\hat{a}=C_{\phi}\left(T_{L}^{\mathrm{T}} \quad T_{n}^{\mathrm{T}}\right)\left[\left(\begin{array}{c}
T_{L} \\
T_{n}
\end{array}\right) C_{\phi}\left(T_{L}^{\mathrm{T}} \quad T_{n}^{\mathrm{T}}\right)\right]^{\dagger}\left(\begin{array}{c}
T_{L} \\
T_{n}
\end{array}\right) a,
$$

where $C_{\phi}$ is the covariance matrix for the turbulence. $C_{\phi}$ is a block diagonal matrix, with the $i$ th block representing the covariance of the $i$ th layer of the atmosphere:

$$
C_{\phi}=\left[\begin{array}{ccccc}
C_{\phi}(1) & & & & 0 \\
& \ddots & & & \\
& & C_{\phi}(i) & & \\
& & & \ddots & \\
0 & & & & C_{\phi}\left(N_{l}\right)
\end{array}\right] \text {, }
$$

where

$$
C_{\phi}(i)=\left\langle a(i) a(i)^{\mathrm{T}}\right\rangle=\gamma(i) C_{L},
$$

$\gamma(i)$ is the strength of layer $i$, and $C_{L}$ is a normalized covariance matrix for a single phase screen with unit strength.

The mean squared difference between the true coefficients of the turbulence, $a$, and their estimate $\hat{a}$ is given by

$$
\begin{aligned}
\left\langle(a-\hat{a})(a-\hat{a})^{\mathrm{T}}\right\rangle= & C_{\phi}-C_{\phi}\left(T_{L}^{\mathrm{T}} \quad T_{n}^{\mathrm{T}}\right) \\
& \times\left[\left(\begin{array}{c}
T_{L} \\
T_{n}
\end{array}\right) C_{\phi}\left(T_{L}^{\mathrm{T}} \quad T_{n}^{\mathrm{T}}\right)\right]^{\dagger}\left(\begin{array}{c}
T_{L} \\
T_{n}
\end{array}\right) C_{\phi},
\end{aligned}
$$

where $C_{\phi}^{\mathrm{T}}=C_{\phi}$ is the covariance of the atmosphere and we have used the identity $A^{\dagger} A A^{\dagger}=A^{\dagger}$.

The wavefront error $\sigma^{2}$ is a weighted sum of the error at each of the $N_{s}$ science points in the FOV:

$$
\sigma^{2}=\sum_{k}^{N_{s}} w_{k}\left\langle\phi_{k}^{2}\right\rangle=\operatorname{Tr}\left[\left\langle\left(b_{s}-b_{m}\right) W\left(b_{s}-b_{m}\right)^{\mathrm{T}}\right\rangle\right],
$$

where Tr is the trace of the matrix and is equal to the sum of the diagonal elements and also to the sum of the eigenvalues. $\phi_{k}$ is the wavefront error at the $k$ th point in the science field. $W$ is a block diagonal matrix of $N_{s}$ blocks whose elements are $w_{k} I$, where $I$ is an identity matrix of dimension equal to the number of Zernike modes computed for each wavefront. If the entire science field is considered, $w_{k}=1 / N_{s}$ for all $k$ points. If only the on-axis science point is considered, $w_{1}=1$ and $w_{k}=0$ otherwise.

For MOAO, the individual phase corrections in each science direction are given by $b_{m}=\hat{b}_{s}$, and substituting this into Eq. (29) yields

$$
\sigma_{M O A O}^{2}=\operatorname{Tr}\left[\left\langle\left(b_{s}-\hat{b}_{s}\right) W\left(b_{s}-\hat{b}_{s}\right)^{\mathrm{T}}\right\rangle\right] .
$$

If we substitute Eq. (20) into Eq. (30), the weighted mean squared error for a MOAO system is

$$
\sigma_{M O A O}^{2}=\operatorname{Tr}\left[T_{s}^{\mathrm{T}} W T_{s}\left\langle(a-\hat{a})(a-\hat{a})^{\mathrm{T}}\right\rangle\right],
$$

where $\left\langle(a-\hat{a})(a-\hat{a})^{\mathrm{T}}\right\rangle$ is defined in Eq. (28).

For MCAO, the residual phase error is the sum of the error in estimating the wavefront and the error in fitting the estimated modes to the DMs:

$$
\sigma_{M C A O}^{2}=\sigma_{e s t}^{2}+\sigma_{f i t}^{2} .
$$

The optimal choice of Zernike coefficients, $m_{*}$, to apply to the DMs to minimize the weighted difference between the estimated wavefront modes $\hat{b}_{s}$ and the correction applied to the mirrors projected to the aperture plane, $b_{m}$ $=T_{m} m$, is given by

$$
\begin{aligned}
m_{*} & =\underset{m}{\arg \min _{m}}=\left(\hat{b}_{s}-b_{m}\right)^{\mathrm{T}} W\left(\hat{b}_{s}-b_{m}\right) \\
& =\underset{m}{\arg \min _{m}}=\left(T_{s} \hat{a}-T_{m} m\right)^{\mathrm{T}} W\left(T_{s} \hat{a}-T_{m} m\right) .
\end{aligned}
$$

By differentiating Eq. (34) with respect to $\mathrm{m}^{\mathrm{T}}$, setting to zero, and solving for $m$, we obtain the optimal coefficients to apply to the DMs, $m_{*}$ :

$$
\begin{aligned}
m_{*} & =\left(T_{m}{ }^{\mathrm{T}} W T_{m}\right)^{-1} T_{m}{ }^{\mathrm{T}} W T_{s} \hat{a} \\
& =P_{m} \hat{a},
\end{aligned}
$$

where $P_{m}=\left(T_{m}{ }^{\mathrm{T}} W T_{m}\right)^{-1} T_{m}{ }^{\mathrm{T}} W T_{s}$. 
The MCAO error is the difference between the true wavefront modes and the optimal correction applied to the DM and projected to the aperture plane:

$$
\begin{aligned}
b_{s}-b_{m_{*}} & =T_{s} a-T_{m} m_{*} \\
& =\left(T_{s}-T_{m} P_{m}\right) a+T_{m} P_{m}(a-\hat{a}) .
\end{aligned}
$$

The weighted mean squared MCAO wavefront error is therefore given by

$$
\begin{aligned}
\sigma_{M C A O}^{2}= & \operatorname{Tr}\left[\left(b_{s}-b_{m_{*}}\right)^{\mathrm{T}} W\left(b_{s}-b_{m_{*}}\right)\right] \\
= & \operatorname{Tr}\left[\left(T_{s}-T_{m} P_{m}\right)^{\mathrm{T}} W\left(T_{s}-T_{m} P_{m}\right) C_{\phi}\right. \\
& \left.+\left(T_{m} P_{m}\right)^{\mathrm{T}} W\left(T_{m} P_{m}\right)\left\langle(a-\hat{a})(a-\hat{a})^{\mathrm{T}}\right\rangle\right],
\end{aligned}
$$

where the second equality follows because

$$
\left(T_{s}-T_{m} P_{m}\right)^{\mathrm{T}} W T_{m} P_{m}=0
$$

and, as before, $\left\langle(a-\hat{a})(a-\hat{a})^{\mathrm{T}}\right\rangle$ is defined in Eq. (28). We may therefore define the fitting and estimation errors for MCAO as the first and second terms in Eq. (40), respectively, such that

$$
\begin{aligned}
\sigma_{f i t}^{2} & =\operatorname{Tr}\left[\left(T_{s}-T_{m} P_{m}\right)^{\mathrm{T}} W\left(T_{s}-T_{m} P_{m}\right) C_{\phi}\right], \\
\sigma_{\text {est }}^{2} & =\operatorname{Tr}\left[\left(T_{m} P_{m}\right)^{\mathrm{T}} W\left(T_{m} P_{m}\right)\left\langle(a-\hat{a})(a-\hat{a})^{\mathrm{T}}\right\rangle\right] .
\end{aligned}
$$

\section{Outer Scale Computation}

It has been noted that as the telescope diameter increases, the performance of the AO system is more likely to be influenced by the outer scale of the atmosphere, $L_{0} \cdot{ }^{17}$ Since we are considering a $30 \mathrm{~m}$ diameter telescope, we therefore need to include the effects of a finite outer scale on the covariance matrix of the atmosphere, $C_{\phi}$. For an infinite $L_{0}$, Noll defines the covariance of the atmosphere in terms of the Zernikes, $a$, as ${ }^{9}$

$$
\begin{aligned}
\left\langle a_{n m}^{*} a_{n^{\prime} m^{\prime}}\right\rangle= & (0.046 / \pi)\left(R / r_{0}\right)^{5 / 3}\left[(n+1)\left(n^{\prime}+1\right)\right]^{1 / 2} \\
& \times(-1)^{\left(n+n^{\prime}-2 n\right) / 2} \delta_{m m^{\prime}} I_{n n^{\prime}}
\end{aligned}
$$

where

$$
I_{n n^{\prime}}=\int_{0}^{\infty} k^{-8 / 3} \frac{J_{n+1}(2 \pi k) J_{n^{\prime}+1}(2 \pi k)}{k^{2}} \mathrm{~d} k,
$$

and $J_{n}$ is an $n$th order Bessel function of the first kind, $n$ and $m$ are the radial and azimuthal order of the Zernikes, respectively, and $k$ is the coordinate in Fourier space. Inclusion of the von Kármán spectrum in Eq. (45) yields

$$
I_{n n^{\prime}}=\int_{0}^{\infty} k^{-1} \frac{J_{n+1}(2 \pi k) J_{n^{\prime}+1}(2 \pi k)}{\left[k^{2}+\left(\frac{R}{L_{0}}\right)^{2}\right]^{11 / 6}} \mathrm{~d} k,
$$

where $R$ is the telescope radius. By using the substitution $\tan \beta=2 \pi k$ in Eq. (46), we obtain

$$
I_{n n^{\prime}}=\int_{0}^{\pi / 2} \frac{\sec ^{2} \beta}{\tan \beta} \frac{J_{n+1}(\tan \beta) J_{n^{\prime}+1}(\tan \beta)}{\left[\left(\frac{\tan \beta}{2 \pi}\right)^{2}+\left(\frac{R}{L_{0}}\right)^{2}\right]^{11 / 6}} \mathrm{~d} \beta .
$$

In this paper, the covariance of the atmosphere is then found by integrating Eq. (47) numerically and substituting $I_{n n^{\prime}}$ into Eq. (44). This method generates diagonal terms with $n=n^{\prime}$, in agreement with those obtained by Winker. ${ }^{18}$ In particular, the effect of the finite outer scale is to attenuate low order Zernike variances.

\section{MONTE CARLO SIMULATIONS}

The method outlined in Section 2 for determining the wavefront error is simulated for different NGS constellations. The NGS constellations are generated randomly within a circular field of diameter 1 or 2 arc min.

Two different modes of NGS operation are considered. First, the NGSs estimate only the first radial order modes (e.g., TT). Only one subaperture is required to estimate these modes. In the second mode of operation, the NGSs estimate first and second radial order modes (e.g., TT, focus, $90^{\circ}$ astigmatism, and $45^{\circ}$ astigmatism). This requires $2 \times 2$ subapertures for a Shack-Hartmann sensor or, equivalently, $2 \times 2$ pixels per image for the pyramid sensor. $^{19}$

When operating with TT NGSs, the simulated system is assumed to have only three available NGS wavefront sensors. Therefore when more than three NGSs appear in the field, the combination of three NGSs that gives the smallest wavefront error is chosen. In these simulations, the optimal combination of three NGSs is found by evaluating all possible combinations of three NGSs. A more practical algorithm to estimate the best available combination of NGSs from the magnitude and position of the NGSs in real time will be required for an actual AO system.

When operating with TTFA NGSs, only one NGS wavefront sensor is assumed. If there is more than one NGS in the field, the best NGS is chosen as the NGS closest to on axis. In a real system, the best TTFA NGS will be a function of star magnitude as well as position.

\section{A. Simulation Parameters}

The system parameters and their values used in these simulations are summarized in Table 1. In addition, the discrete atmospheric turbulence profile typical of Cerro Pachon-and which is used in the simulations-is tabulated in Table $2 .^{20}$ Two modes of operation are considered:

Table 1. System Parameters Used in This Paper

\begin{tabular}{lcc}
\hline \multicolumn{1}{c}{ Parameter } & Symbol & Value \\
\hline Telescope diameter & $D$ & $30 \mathrm{~m}$ \\
Fried's parameter $^{a}$ & $r_{0}$ & $0.15 \mathrm{~m}$ \\
Evaluation wavelength & $\lambda$ & $0.5 \mu \mathrm{m}$ \\
Height of sodium layer & $H$ & $90 \mathrm{~km}$ \\
DM conjugate altitudes & $h_{m}$ & $010 \mathrm{~km}$ \\
Zernike radial order & $N$ & 6 \\
\hline
\end{tabular}

${ }^{a}$ Ref. 21. 
Table 2. Six-Layer Turbulence Profile, Typical of Cerro Pachon, ${ }^{a}$ Showing the Height $h(i)$ of Each Layer and the Relative Turbulence Strength $\gamma(i)$

\begin{tabular}{crc}
\hline Layer $i$ & $h(i)(\mathrm{m})$ & $\gamma(i)$ \\
\hline 1 & 0 & 0.6523 \\
2 & 2577 & 0.1723 \\
3 & 5155 & 0.0551 \\
4 & 7732 & 0.0248 \\
5 & 12887 & 0.0736 \\
6 & 15464 & 0.0219 \\
\hline
\end{tabular}

${ }^{a}$ Ref. 20.

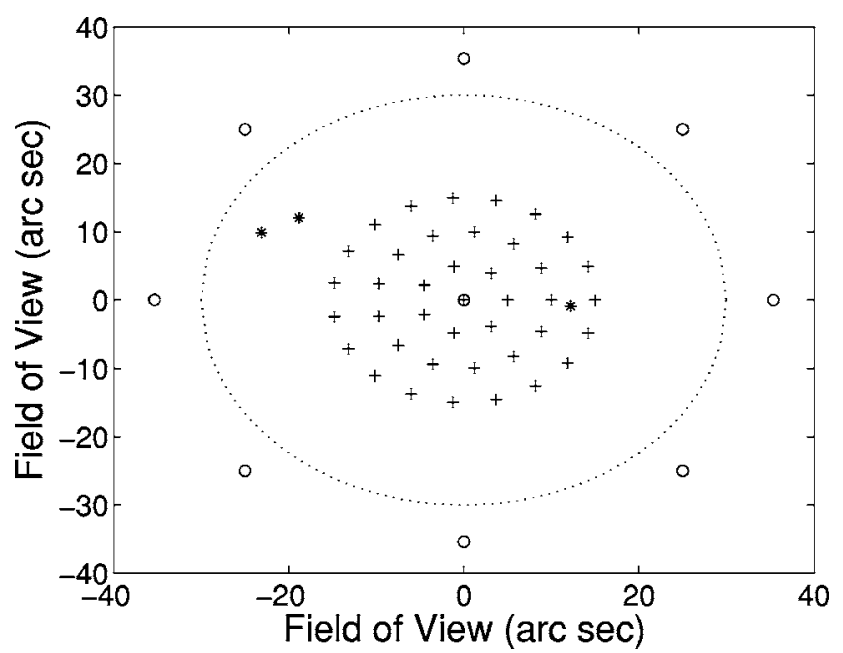

Fig. 1. Example plot of the FOV, where there are eight LGSs (circles) regularly arranged on a circle of diameter $50 \sqrt{2}$ arc sec and one on-axis LGS, three randomly distributed NGSs (asterisks) bounded within a 1 arc min diameter field (dotted line), and a science field of 40 points located within a circle of diameter 30 arc sec (plusses).

the on-axis performance of MOAO and the performance of MCAO averaged over an extended FOV. For MOAO, there is one DM for the on-axis object, which is taken to be conjugate to the ground layer $(0 \mathrm{~km})$. This MOAO configuration is therefore equivalent to a single-conjugate, narrowfield AO system. For MCAO, there are two DMs, which are chosen to be conjugate to 0 and $10 \mathrm{~km}$.

The number of LGSs in the asterism, $N_{L G S}$, is chosen to be 5,7 , or 9 . The LGS asterism investigated consists of one on-axis LGS and $N_{L G S}-1$ LGSs equally separated on a ring of diameter $50 \sqrt{2}$ arc sec. The LGS asterism with $N_{L G S}=9$ is shown in Fig. 1. Initial simulation tests have shown that this regular LGS asterism is not optimal and that introducing a measure of randomness to break the regularity of the ring can improve the overall mean squared wavefront error by approximately $15 \%$. However, the LGS asterisms are not the subject of this paper and are not investigated further. The science FOV is defined as 40 points arranged in concentric rings within a 30 arc sec diameter circle, as shown in Fig. 1.

We are primarily interested in the wavefront error due to the low order modes (the first three radial orders), since these are the modes that the NGS can help us estimate. Figure 2 shows the wavefront error versus the ra- dial order of Zernikes considered, where all orders are evaluated to find the wavefront error (solid curve), and only the first three orders are evaluated to find the wavefront error (dashed curve). At six radial orders, the difference between the two curves is small (only a $3 \%$ difference), showing that most of the wavefront error is due to the low order modes. We choose the number of radial orders of the Zernikes, $N$, for the simulations to be six, which corresponds to the first 27 Zernike polynomials, excluding piston, to allow fast computation. In the simulations, all six radial orders considered are also evaluated. For the trade-offs associated with sky coverage, such as NGS field diameter or choice of which Zernike modes to estimate with the NGS, it is therefore sufficient to consider the low order terms, since the higher order terms do not couple strongly into the lower order terms. For a full error budget, however, it would be necessary to include more Zernike modes or to add in the higher order terms obtained from time-domain simulations.

The simulation parameter space, i.e., the parameters that are varied in the simulations and the different values investigated, is shown in Table 3.

\section{B. Simulation Results}

Each case in the simulation parameter space is run over 1000 different random NGS configurations. A single

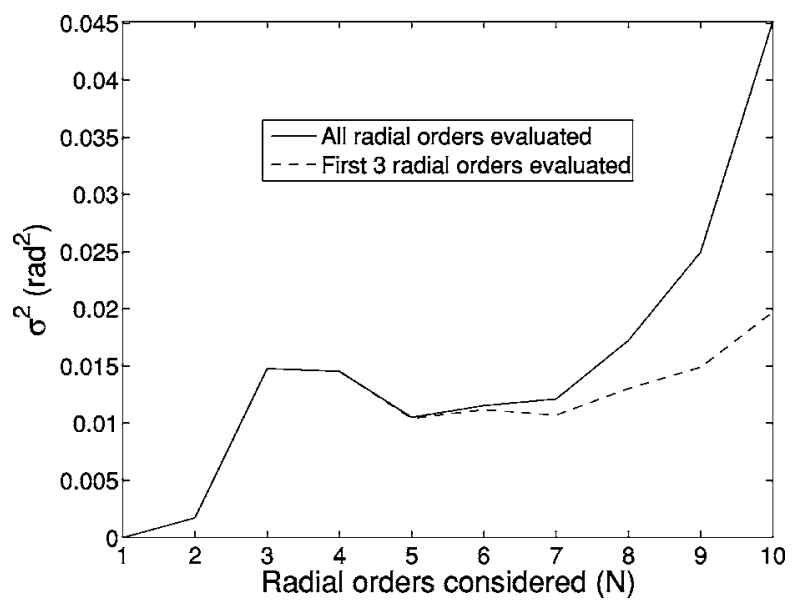

Fig. 2. Wavefront error versus number of radial orders of Zernikes considered, where all the Zernike modes that are considered are evaluated (solid curve) and only the first three radial modes are evaluated (dashed curve). There are three NGSs that measure TT, which are located in an equilateral triangle whose vertices are situated on a circle of diameter 15 arc sec. The system is operating in MOAO narrow-field mode, with $N_{L G S}=7$ and $L_{0}=\infty$.

Table 3. Parameter Space Investigated in the Simulations

\begin{tabular}{lcc}
\hline \multicolumn{1}{c}{ Parameter } & Symbol & Values \\
\hline Mode of operation & - & MOAO narrow field, \\
& & MCAO wide field \\
NGS field diameter & - & 1,2 (arc min) \\
Outer scale & $L_{0}$ & $30,60, \infty,(\mathrm{m})$ \\
Number of LGSs & $N_{L G S}$ & $5,7,9$ \\
NGS modes & - & TT, TTFA \\
\hline
\end{tabular}



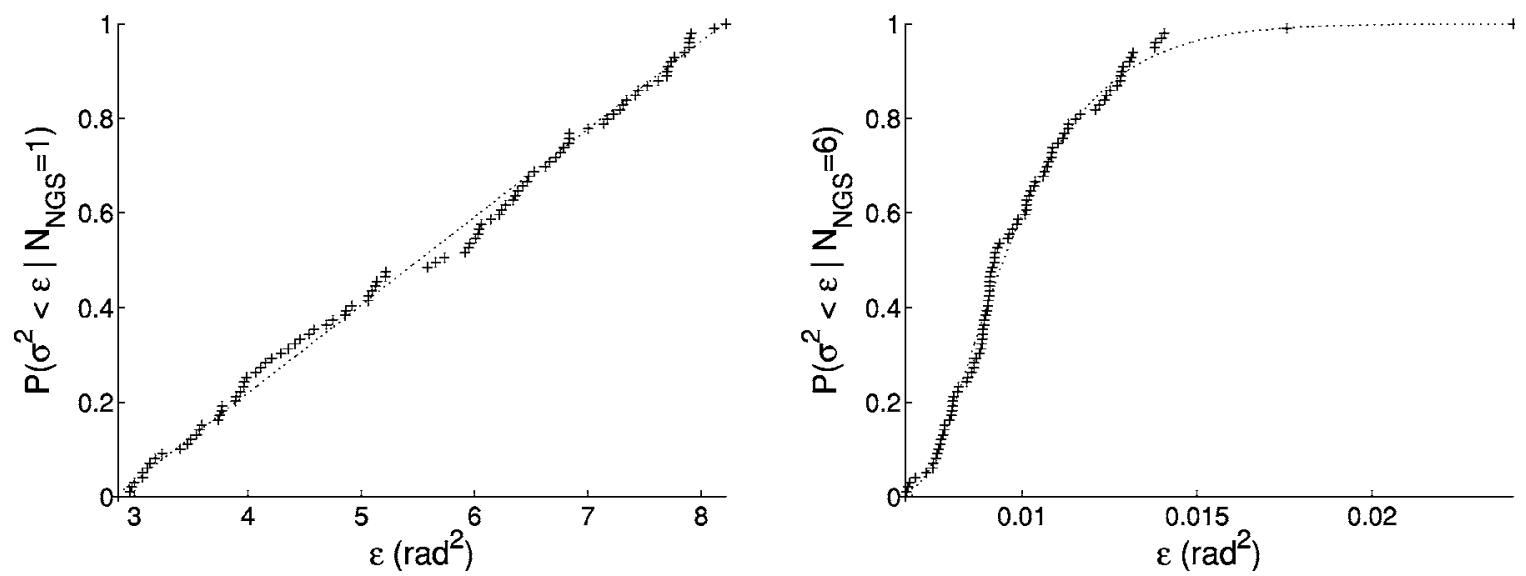

Fig. 3. Example plots of $\operatorname{Pr}\left(\sigma^{2}<\epsilon \mid N_{N G S}=m\right)$ for (a) $m=1$ and (b) $m=6$. The plusses are the values obtained from a random selection of 100 out of the 1000 Monte Carlo simulations, and the dotted curves are the best fit curves to the 1000 points.

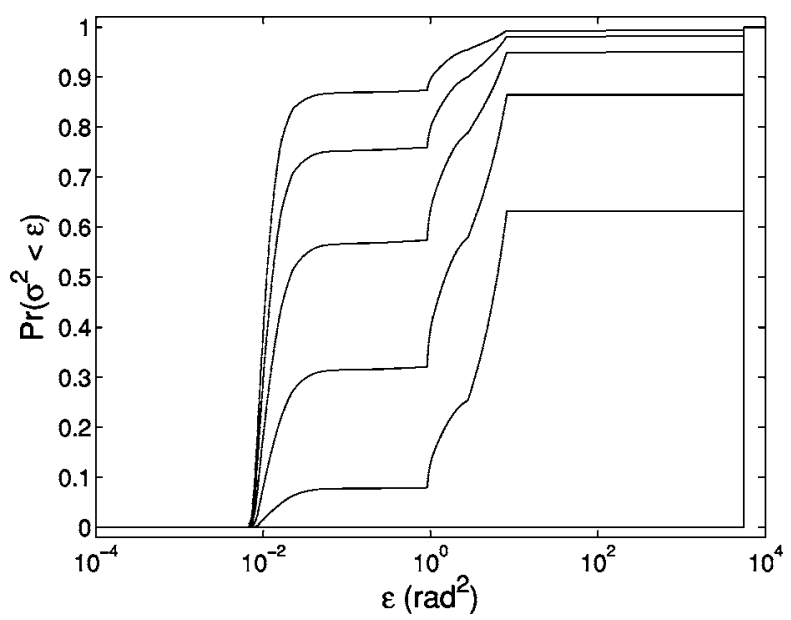

Fig. 4. Example plot of $\operatorname{Pr}\left(\sigma^{2}<\epsilon\right)$ versus $\epsilon$ for $\mu_{N G S}=0,1,2,3,4$, and 5 arc $\mathrm{min}^{-2}$, where $\mu_{N G S}=0$ arc $\mathrm{min}^{-2}$ is the bottom curve, $\mu_{N G S}=1 \mathrm{arc} \mathrm{min}^{-2}$ is the second to bottom, and so on.

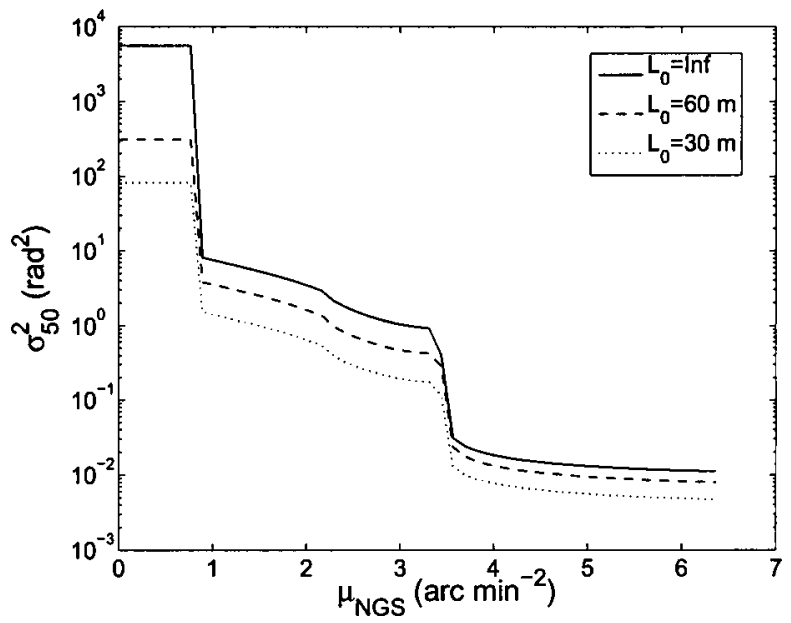

Fig. 5. Median error $\sigma_{50}^{2}$ versus mean NGS density in the field, $\mu_{N G S}\left(\operatorname{arc~} \min ^{-2}\right)$, for $L_{0}=\infty$ (solid curve), $L_{0}=60 \mathrm{~m}$ (dashed curve), and $L_{0}=30 \mathrm{~m}$ (dotted curve). The system is running in MOAO narrow-field mode, with seven LGSs, the NGSs measure TT only, and the NGS guide field diameter is 1 arc min.
Monte Carlo simulation of one case in the simulation parameter space takes on the order of $0.3 \mathrm{~s}$.

We define the probability distribution for $N_{N G S}$ NGSs $\left(\operatorname{arc} \min ^{-2}\right)$ in the FOV as a Poisson distribution with a mean of $\mu_{N G S}$ NGSs $\left(\operatorname{arc} \min ^{-2}\right)$, given by

$$
\operatorname{Pr}\left(N_{N G S}=m\right)=\frac{\exp \left(-\mu_{N G S}\right)\left(\mu_{N G S}\right)^{m}}{m !},
$$

where $\operatorname{Pr}(\cdot)$ denotes probability and ! is the factorial operator. The $m$ NGSs are randomly selected within a circular FOV of diameter 1 or 2 arc min.

A cumulative density function $(\mathrm{CDF}), \operatorname{Pr}\left(\sigma^{2}<\epsilon \mid N_{N G S}\right.$ $=m)$, that the error is less than a threshold $\epsilon\left(\operatorname{rad}^{2}\right)$ for a given number $m$ of NGSs in the field is plotted from the 1000 simulation points and fitted as a sum of exponentials. These CDFs are generated for $m=0$ to 6 . The probability of there being more than six NGSs in the field is negligible. Example CDFs for $m=1$ and $m=6$ and simulation parameters (MOAO, TT NGS, $N_{L G S}=7,1$ arc sec guide field, and $L_{0}=\infty$ ) are shown in Fig. 3 .

The probability that the error is less than the threshold for a mean of $\mu_{N G S}$ NGSs $\left(\operatorname{arc~} \min ^{-2}\right)$ is given by

$$
\operatorname{Pr}\left(\sigma^{2}<\epsilon\right)=\sum_{m=0}^{6} \operatorname{Pr}\left(N_{N G S}=m\right) \operatorname{Pr}\left(\sigma^{2}<\epsilon \mid N_{N G S}=m\right),
$$

where | denotes conditional probability, $\operatorname{Pr}\left(N_{N G S}=m\right)$ is found by using Eq. (48), and $\operatorname{Pr}\left(\sigma^{2}<\epsilon \mid N_{N G S}=m\right)$ is curve fitted from the simulations as described previously. An example CDF, plotted for several different values of $\mu_{N G S}$, and simulation parameters (MOAO, TT NGS, $N_{L G S}=7$, 1 arc sec guide field, and $\left.L_{0}=\infty\right)$ is shown in Fig. 4 . It is apparent from this graph that the wavefront error is significantly reduced to $0.1 \mathrm{rad}^{2}$ or less when there are three or more TT NGSs in the field.

It is important to note the piecewise nature of the CDFs plotted in Fig. 4. These "steps" arise due to the vastly different $\epsilon$ scales for $N_{N G S}=0,1,2$, and 3 , which means that when added in Eq. (49) the individual CDFs do not overlap. The median error $\sigma_{50}^{2}\left(\operatorname{rad}^{2}\right)$ for a given $\mu_{N G S}$ is found from the graph of $\operatorname{Pr}\left(\sigma^{2}<\epsilon\right)$ versus $\epsilon$ (e.g., Fig. 4) as the abscissa corresponding to $\operatorname{Pr}\left(\sigma^{2}<\epsilon\right)$. 
The median error $\sigma_{50}^{2}$ is then plotted versus $\mu_{N G S}$ for each of the parameters in the simulation parameter space in Figs. 5-9 to give an estimate of sky coverage for the different simulation parameters. Figures 5-9 all show a stepped response, which is due to the piecewise nature of the $\operatorname{Pr}\left(\sigma^{2}<\epsilon\right)$ versus $\epsilon$ curves described previously.

As can be seen in Fig. 5, the inclusion of the finite outer scale significantly reduces the median wavefront error for all mean densities of NGS in the FOV. This is not surprising, since inclusion of the finite outer scale reduces the covariance of the low order terms, which contain most of the energy of the wavefront.

Figure 6 shows the improvement gained with an increased number of LGSs in the asterism. This improvement is significant only when there are three or more NGSs in the field.

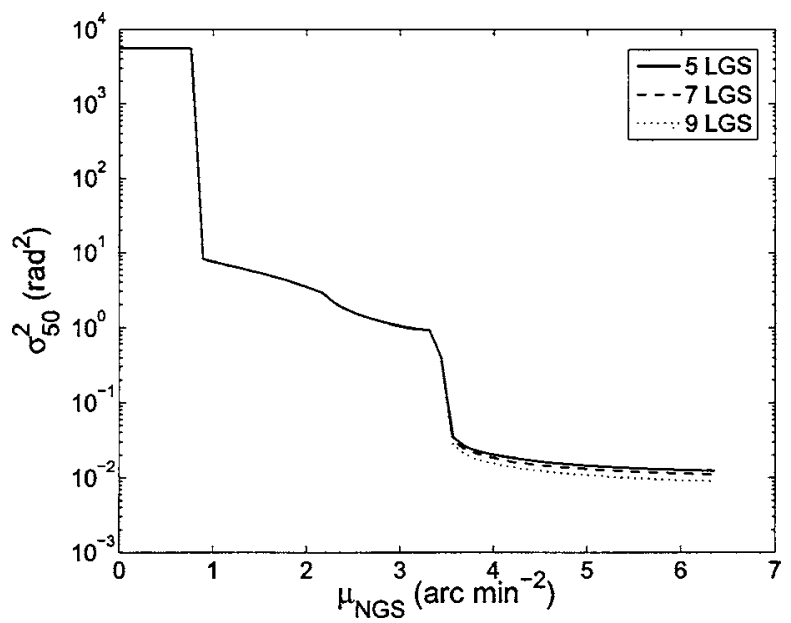

Fig. 6. Median error $\sigma_{50}^{2}$ versus mean NGS density in the field, $\mu_{N G S}\left(\operatorname{arc} \min ^{-2}\right.$ ), for $N_{L G S}=5$ (solid curve), $N_{L G S}=7$ (dashed curve), and $N_{L G S}=9$ (dotted curve) LGSs, respectively. The system is running in MOAO narrow-field mode, with $L_{0}=\infty \mathrm{m}$, the NGSs measure TT only, and the NGS guide field diameter is 1 arc min.

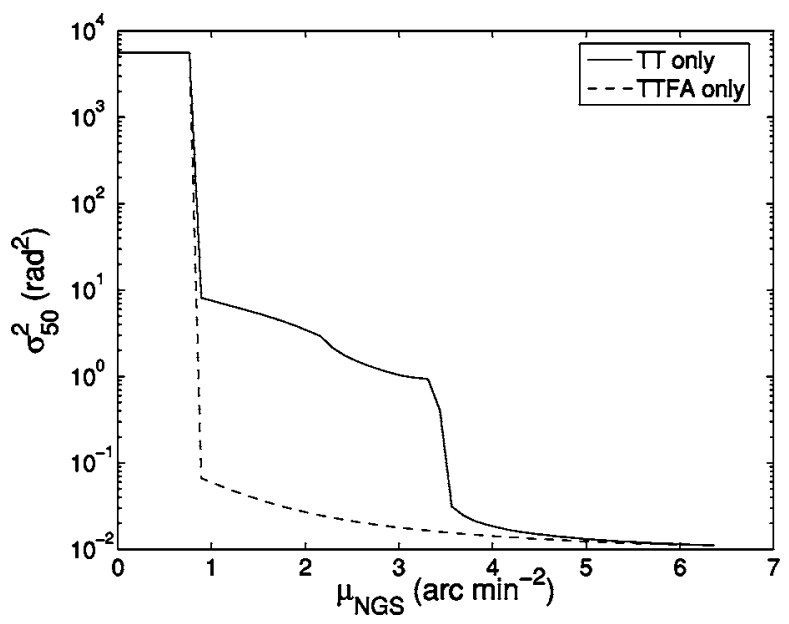

Fig. 7. Median error $\sigma_{50}^{2}$ versus mean NGS density in the field, $\mu_{N G S}\left(\operatorname{arc} \min ^{-2}\right)$, for up to three NGSs measuring TT modes only (solid curve) and a single NGS measuring TTFA modes only (dashed curve). The system is running in MOAO narrow-field mode, with $N_{L G S}=7, L_{0}=\infty$, and the NGS guide field diameter is 1 arc min.

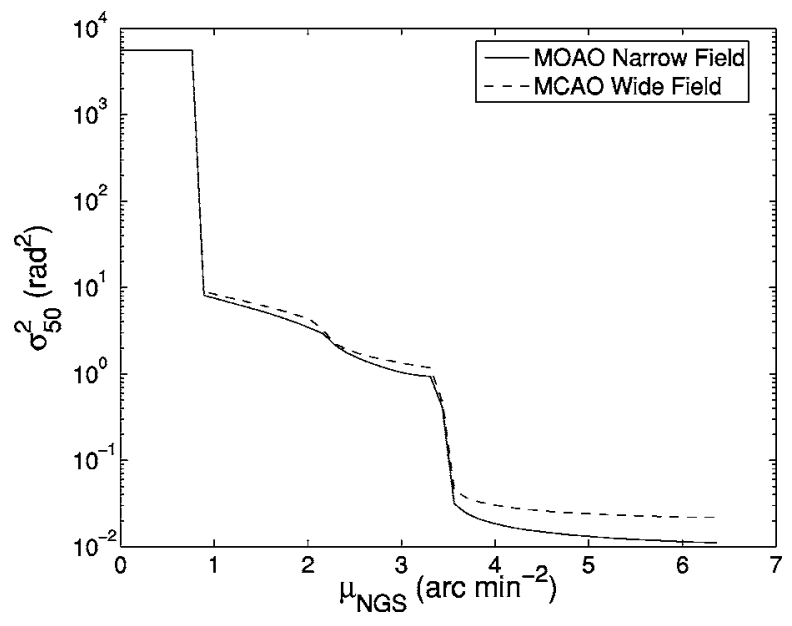

Fig. 8. Median error $\sigma_{50}^{2}$ versus mean NGS density in the field, $\mu_{N G S}\left(\operatorname{arc} \min ^{-2}\right)$, for MOAO narrow field-mode (solid curve) and MCAO wide-field mode (dashed curve). The system has $N_{L G S}=7$ and $L_{0}=\infty$, the NGSs measure TT only, and the NGS guide field diameter is 1 arc min.

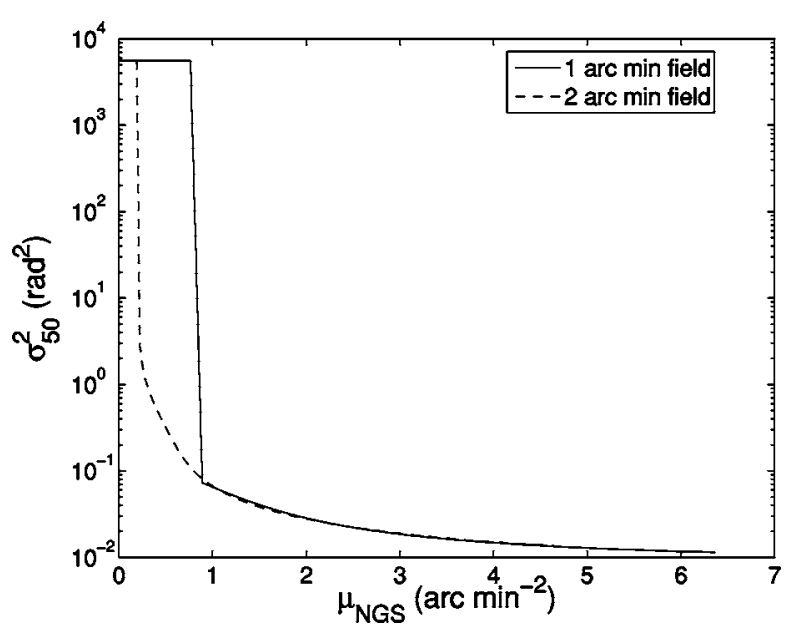

Fig. 9. Median error $\sigma_{50}^{2}$ versus mean NGS density in the field, $\mu_{N G S}\left(\operatorname{arc~} \min ^{-2}\right)$, for either a 1 arc min diameter NGS field (solid curve) or a 2 arc min diameter NGS field (dashed curve). The system is running in MOAO narrow-field mode, with $N_{L G S}=7$ and $L_{0}=\infty$, and a NGS that measures TTFA only.

The difference between using TT and TTFA NGSs is shown in Fig. 7. Clearly, when there are only a few NGSs in the FOV (1-3 NGS arc min $^{-2}$ ), using TTFA NGSs yields a far lower median wavefront error than that moving TT NGS. However, it should be noted that in practice a brighter star is required to run an AO system using a TTFA NGS than one using a TT NGS, and further study is required to determine the preferred approach. When there are several NGSs in the FOV (more than 3 NGS arc $\min ^{-2}$ ), TT and TTFA NGSs converge to roughly the same level of performance.

As can be seen in Fig. 8, the median error of a narrowfield MOAO system is, as expected, always less than that of a MCAO wide-field system. It should be noted that the sky coverage curves for both have the same shape.

Figure 9 shows the comparison of the median wavefront error with 1 and 2 arc min NGS guide fields. For a mean GS density less than 0.2 NGS arc $\mathrm{min}^{-2}$, the me- 
dian wavefront error corresponds to zero GSs within either the 1 or 2 arc min fields, and the performance of the two NGS field diameters is the same. For a mean GS density between 0.2 and 0.9 NGS $\operatorname{arc} \mathrm{min}^{-2}$, the median wavefront error corresponds to the best NGS being within the 2 arc min field but not within the 1 arc min field. Consequently, the median wavefront error of the 2 arc min field is significantly lower than that of the 1 arc min field. For a mean GS density of more than 0.9 NGS arc $\mathrm{min}^{-2}$, the median wavefront error corresponds to the best NGS being within the 1 arc min field, and thus the performance of the two NGS field diameters is the same. The slight difference between the curves in Fig. 9 for more than $0.9 \mathrm{NGS}$ arc $\mathrm{min}^{-2}$ is due to the finite number of simulations for each number of GSs in the field (1000) and the error in fitting the exponentials to the simulation results.

\section{CONCLUSION}

A new method for calculating the sky coverage of a LGS AO system has been presented. This method takes into account the effects of off-axis and finite altitude GSs, may be used with multiple LGS systems, and allows fast Monte Carlo simulations over random NGS configurations. The Monte Carlo simulations have shown that inclusion of the finite outer scale of the atmosphere significantly reduces the median wavefront error, that the median wavefront error decreases as more LGSs are included in the LGS asterism, that a larger NGS field provides a better median wavefront error at lower star densities in the field, and that a TTFA NGS provides a better median wavefront estimate than that from three TT NGSs.

A number of practical issues need to be incorporated into this model, including the effects of servo lag with a finite closed loop bandwidth, and wavefront sensor noise with stars of finite brightness. These will be addressed in a future paper.

\section{ACKNOWLEDGMENTS}

This paper was prepared as part of the work of the Thirty Meter Telescope (TMT) Project. TMT is a partnership of the Association of Universities for Research in Astronomy (AURA), the Association of Canadian Universities for Research in Astronomy (ACURA), the California Institute of Technology, and the University of California. The partners gratefully acknowledge the support of the Gordon and Betty Moore Foundation, the U.S. National Science Foundation (NSF), the National Research Council of Canada, the Natural Sciences and Engineering Research Council of Canada, and the Gemini Partnership.

The New Initiatives Office is a partnership between two divisions of the AURA; the National Optical Astronomy Observatory (NOAO) and the Gemini Observatory. NOAO is operated by AURA under cooperative agreement with the NSF. The Gemini Observatory is operated by AURA under a cooperative agreement with the NSF on behalf of the Gemini partnership: the NSF (United States), the Particle Physics and Astronomy Re- search Council (United Kingdom), the National Research Council (Canada), CONICYT (Chile), the Australian Research Council (Australia), CNPq (Brazil), and CONICET (Argentina).E-mail: rclare@caltech.edu.

\section{REFERENCES}

1. F. Roddier, "The effect of atmospheric turbulence in optical astronomy," in Progress in Optics, E. Wolf, ed. (NorthHolland, 1981), pp. 283-376.

2. H. W. Babcock, "The possibility of compensating astronomical seeing," Publ. Astron. Soc. Pac. 65, 229-236 (1953).

3. P. L. Wizinowich and D. Bonaccini, eds., Adaptive Optical System Technologies II, Proc. SPIE 4839 (2003).

4. T. Andersen and A. Ardeberg, eds., Extremely Large Telescopes II, Proc. SPIE 5382 (2004).

5. D. B. Calia, B. L. Ellerbroek, and R. Ragazzoni, eds. Advancements in Adaptive Optics, Proc. SPIE 5490 (2004).

6. R. Foy and A. Labeyrie, "Feasibility of adaptive optics with laser probe," Astron. Astrophys. 152, L29-L32 (1985).

7. J. M. Beckers, "Increasing the size of the isoplanatic patch with multiconjugate adaptive optics," in ESO Conference on Very Large Telescopes and Their Instrumentation, M. H. Ulrich, ed. (European Southern Observatory, 1988), Vol. 2, pp. 693-703.

8. F. Assemat, F. Hammer, E. Gendron, F. Sayede, P. Laporte, M. Marteaud, M. Puech, J.-M. Conan, T. Fusco, A. Liotard, and F. Zamkotskian, "FALCON: a new-generation spectrograph with adaptive optics for the ESO VLT," in Optics in Atmospheric Propagation and Adaptive Systems VI, J. D. Gonglewski and K. Stein, eds., Proc. SPIE 5237, 211-222 (2004).

9. R. Noll, "Zernike polynomials and atmospheric turbulence," J. Opt. Soc. Am. 66, 207-211 (1976).

10. R. C. Flicker, F. J. Rigaut, and B. L. Ellerbroek, "Tilt anisoplanatism in laser-guide-star-based multiconjugate adaptive optics," Astron. Astrophys. 400, 1199-1207 (2003).

11. B. L. Ellerbroek and F. R. Rigaut, "Methods for correcting tilt anisoplanatism in laser-guide-star-based multiconjugate adaptive optics," J. Opt. Soc. Am. A 18, 2539-2547 (2001).

12. M. Lloyd-Hart and N. M. Milton, "Fundamental limits on isoplanatic correction with multiconjugate adaptive optics," J. Opt. Soc. Am. A 20, 1949-1957 (2003).

13. R. Ragazzoni, E. Marchetti, and F. Rigaut, "Modal tomography for adaptive optics," Astron. Astrophys. 342, L53-L56 (1999).

14. M. Le Louarn and M. Tallon, "Analysis of modes and behavior of a multiconjugate adaptive optics system," J. Opt. Soc. Am. A 19, 912-925 (2002).

15. T. Fusco, J-M Conan, G. Rousset, L. M. Mugnier, and V. Michau, "Optimal wave-front reconstruction strategies for multiconjugate adaptive optics," J. Opt. Soc. Am. A 18, 2527-2538 (2001)

16. H. L. van Trees, Detection, Estimation, and Modulation Theory (Wiley, 1968).

17. B. L. Ellerbroek, "Including outer scale effects in zonal adaptive optics calculations," Appl. Opt. 36, 9456-9467 (1997).

18. D. M. Winker, "Effect of a finite outer scale on the Zernike decomposition of atmospheric optical turbulence," J. Opt. Soc. Am. A 8, 1568-1573 (1991).

19. R. M. Clare and R. G. Lane, "Wavefront sensing from subdivision of the focal plane with a lenslet array," J. Opt. Soc. Am. A 22, 117-125 (2005).

20. J. Vernin, A. Agabi, R. Avila, M. Azouit, R. Conan, F. Martin, E. Masciadri, L. Sanchez, and A. Ziad, "1998 Gemini site testing campaign: Cerro Pachon and Cerro Tololo," Gemini document RTP-AO-G0094 (Gemini Observatory, 2000).

21. D. L. Fried, "Optical resolution through a randomly inhomogenous medium for very long and very short exposures," J. Opt. Soc. Am. 56, 1376-1379 (1966). 